\title{
Plant signalling and the role played by photosynthates in mycorrhizal recruitment
}

\author{
Thomas PW ${ }^{1}$ and Jones $\mathbf{C L}^{1}$ \\ ${ }^{1}$ Mycorrhizal Systems Ltd, Unit 4a, Popples Close Farm, Heptonstall, HX7 7PG. United Kingdom. \\ Paul@Mycorrhizalsystems.com
}

Thomas PW, Jones CL 2013 - Plant signalling and the role played by photosynthates in mycorrhizal recruitment. Mycosphere 4(5), 952-958, Doi 10.5943/mycosphere/4/5/8

\begin{abstract}
Previous research into the recruitment and regulation of ectomycorrhizal partnerships have found both secondary compounds such as IAA and hypaphorine and the physical limitation of nutrient exchange to play important roles. In this study, Quercus robur seedlings displaying a normalized growth pattern and mutant individuals lacking photosynthetic apparatus were inoculated with a sporal treatment of Tuber aestivum in order to elucidate a role for photosyntheticly induced signals in mycorrhizal recruitment. We found that Quercus robur that lacked an above-ground stem system and therefore the apparatus for photosynthesis recruited no Tuber aestivum, while those with a stem complex showed normal-high mycorrhizal recruitment therefore leading to the conclusion that photosynthates and photosynthetically induced signalling compounds such as phytohormones play an important role within the initial recruitment of mycorrhiza to plants.
\end{abstract}

Key words - ectomycorrhiza - hypaphorine -IAA - mutualism - Photosynthates - Quercus robur - Tuber aestivum syn - uncinatum

\section{Introduction}

Around $80 \%$ of all recorded plant species form root associations with fungi known as mycorrhiza (Wang \& Qiu 2006), among which are the most valuable and highly prized fungi in the world. These associations are based upon the mutual exchange of resources, with the mycorrhiza providing the plant with nitrogen and minerals and the plant providing the mycorrhiza with sugars (Lambers et al. 1998).

Mycorrhizal associations can be categorized into two groupings and are termed either endoor ectomycorrhiza. Endo- and ectomycorrhiza differ in their form of root attachment. Endomycorrhiza are obligate fungal mutualists in which a large percentage of the fungal tissue is contained within the plants' root cortical cells. Ectomycorrhiza are facultative fungal mutualists in which a large percentage of the fungal tissue is found outside of the root cortical cells (Lambers et al. 1998). Although the details of this mutualism have been documented within a number of disciplines from ecology and mycology to biochemistry and cell biology, further research is required to fully understand the relationship between fungal partners and their plant hosts.

In ectomycorrhizal (ECM) associations, due to a larger portion of the fungal tissue being contained outside of the root cortical cell, there is no direct symplastic pathway between the two partners. This means that the nutrients being exchanged must pass through an apoplastic interface 
before either partner can absorb them (Peterson \& Bonfante 1994). This interface is called the Hartig net and is a pseudoparenchymatous sheath enveloping the developing root nodules. It contains intercellular hyphae linking the epidermal and cortical cells, across which apoplastic exchange may take place (Smith et al. 1994).

The establishment of the mycorrhiza is accompanied by several morphological root changes induced by the interaction of two normally antagonistic hormones produced by both the fungal and plant partners (Martin et al. 1987, Barker et al. 1998, Ditengou \& Lapeyrie 2000, Reboutier et al. 2002). The morphological changes to the root structure involve the reduction of tap root growth, the stimulation of lateral root growth, changes to epidermal cell shape and inhibition of root hair elongation (Martin et al. 1987, Barker et al. 1998). The roles of the antagonistic phytohormones indole-3-acetic acid (IAA) and hypaphorine are the subject of contention with regards to mycorrhizal association and its subsequent changes in root morphology. Work on hyper IAAproducing plant mutants has found that increased IAA production leads to increased changes in root morphology towards those found in ECM associations. It was concluded that fungal-derived IAA does not seem to be directly involved in host plant growth stimulation by ectomycorrhizal fungi (Gay et al. 1994). Hypaphorine (tryptophan betaine), which is competitively antagonistic towards IAA (Gay et al. 1994, Reboutier et al. 2002) is the first fungal molecule that induces differentiation for the symbiosis (Béguiristain et al. 1995). Hypaphorine acts on the root morphology, in particular inhibiting root hair elongation and altering the cytoskeleton. IAA restores this elongation, a discovery which first led to the hypothesis of IAA and hypaphorine antagonism (Ditengou et al. 2003).

From research it is clear that the two phytohormones are both present at the time of association and have distinct morphological effects on root morphology as well as antagonistic effects on one another. However, current findings to date have not conclusively found which of the two primary compounds is acting upon the association. Nor is it clear whether the antagonistic effects of the two are required to effect and control the morphological changes required for ectomycorrhizal attachment to the root.

Some level of regulation of the fungal partner has been found in plants with ectomycorrhizal associations; however, it is the control of carbohydrates as well as the use of costly secondary compounds which regulates this relationship (Son \& Smith 1988, Regvar \& Gogola 1996, Bücking \& Heyser 2003). Carbohydrate compounds may only be exchanged with the mycorrhiza when the plant can spare them, the main limiting factor to carbohydrate levels in plants being rates of photosynthesis. Any reduction in carbohydrate exchange from the plant automatically leads to a reduction in nutrient exchange from the mycorrhiza. This regulates the relationship and allows either partner to exchange only what they can spare or can expect to receive reciprocation for. The most heavily researched group with regards to the regulation and energetic costs of the mutualism are the endomycorrhiza. However, the effects of photosynthesis limitation have also been documented with ectomycorrhizal associations. Higher competition for carbohydrates from both partners has been found to cause a decrease in P inflow and root colonisation (Son \& Smith1988). The availability of photosynthates for the fungal partner has also been shown to reduce when the plant is under low light conditions (Bücking \& Heyser 2003).

In this investigation we aim to examine the effects of light conditions on the recruitment of the ectomycorrhiza Tuber aestivum (summer truffle) to the plant symbiont Quercus robur using healthy trees with well developed stem systems and mutant individuals displaying no above-ground growth. We intend to practically demonstrate the relationship between irradiance (and therefore photosynthates) and ectomycorrhizal recruitment. We will examine whether the plants are able to signal to the roots the availability of photosynthetically produced sugars and the possible mechanisms/compounds involved in this signalling. Both sets of individuals have well developed root systems and are being measured after one year of growth from germination. Therefore, all of the trees in the study have some level of sugar storage in their roots and seed-reserves. 


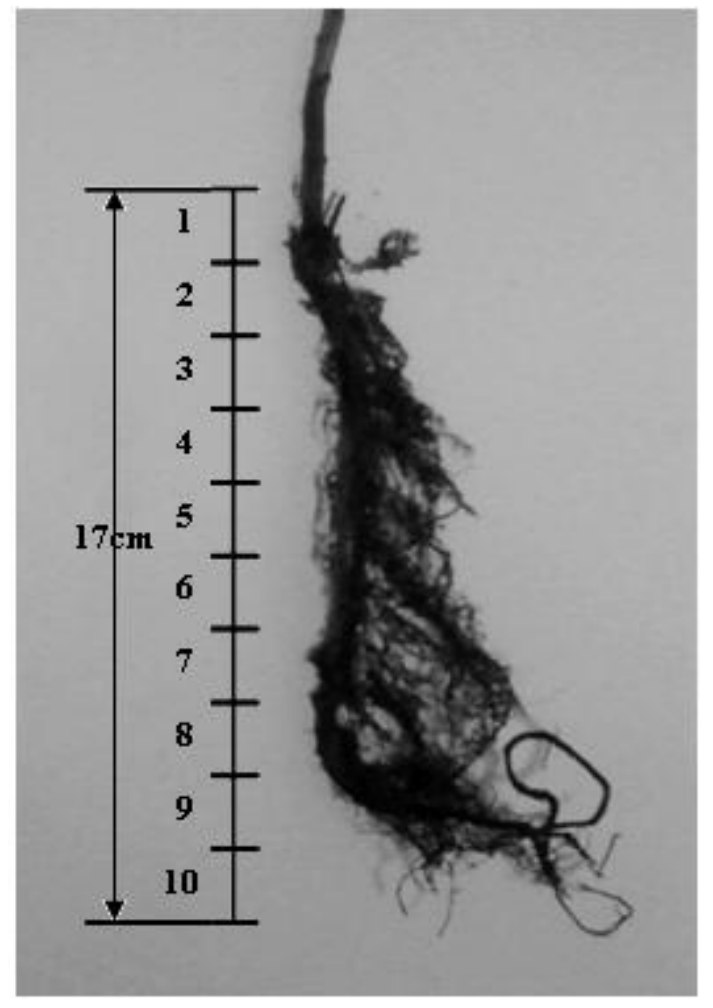

Fig. 1 - Diagram of root division system. Root system divisions showing the 10 sections including lateral roots, each root section measuring $1.7 \mathrm{~cm}$.

\section{Materials \& Methods}

\section{Cultivating trees and sampling root sections}

1000 Quercus robur saplings were grown from seed in sterile substrate and inoculated with a standard sporal treatment for Tuber aestivum syn uncinatum. Thirty trees with above ground stem systems and 30 without were randomly selected at the end of the growing season (September) after one years' growth. Their root systems were then divided into 10 equal sections, including all lateral roots, and numbered (Fig. 1). A random number generator was used to select a root section from each tree to be measured.

\section{Measuring colonisation}

Forty tips from each randomly selected root section were studied under a dissecting microscope for mycorrhizal colonisation. The number of root tips colonised of the 40 examined was then used to calculate a percentage colonisation for each root section.

\section{DNA testing}

Spot checking with PCR analysis confirmed the presence of Tuber aestivum syn. uncinatum within the samples, with successful PCR amplification. Samples tested at Linnaeus viral and plant diagnostic laboratories, Gisborne, New Zealand.

\section{Statistical Analysis}

Stem length and depth in profile were tested for normality using Shapiro Wilk, $w=0.89, \mathrm{n}=$ $30, p>0.5$. These data were not normal. A Spearman's Rank was used to measure correlations between stem length and percentage colonisation and depth in profile and percentage colonisation, to examine possible limiting factors beside irradiance that might influence levels of mycorrhizal colonisation. The data detailing the percentage colonisation were tested for normality using Shapiro Wilk, $\mathrm{w}=0.88, \mathrm{n}=10, \mathrm{p}=0.01$. These data were normal. A Student's $\mathrm{t}$ test was then used to test for any significant difference in colonisation between the two treatments. 


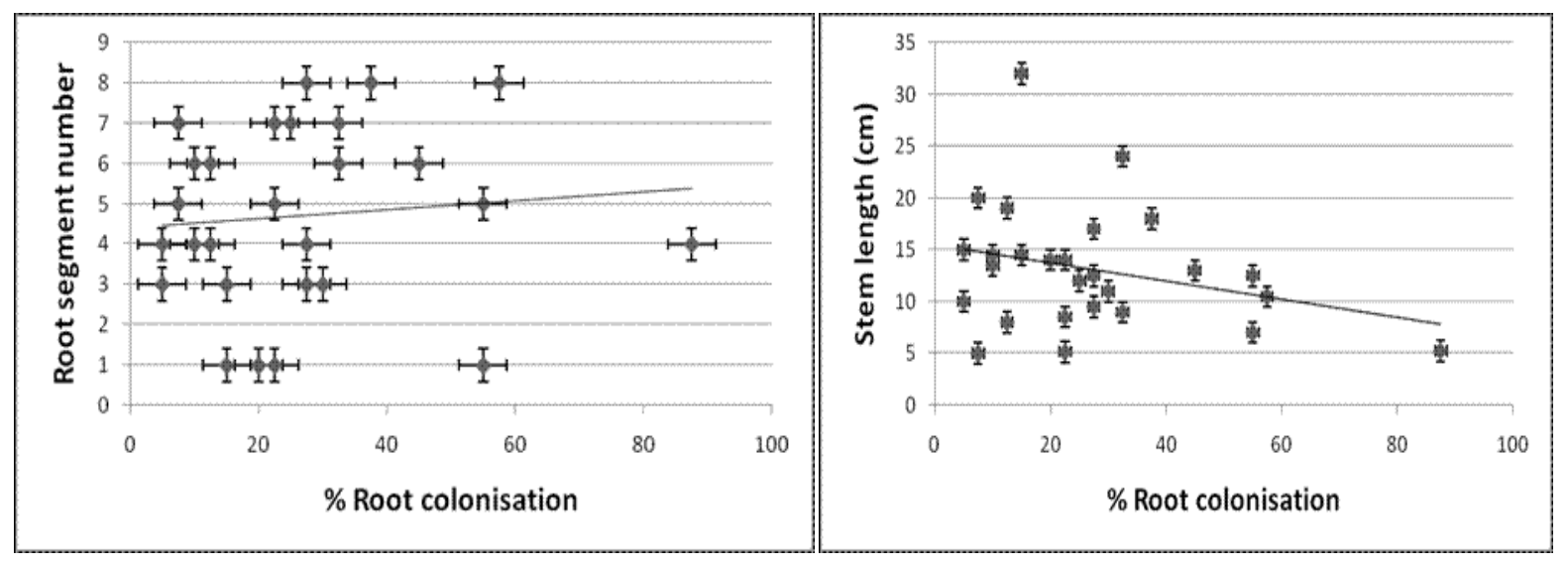

Fig. 2 - Relationship between \% mycorrhizal colonisation and tree physiology. A. The relationship between root section number and \% root colonisation $( \pm 1$ s.e.m.) showing no significant correlation. B. The relationship between stem length and \% root colonisation $( \pm 1$ s.e.m. $)$ showing no significant correlation.

\section{Results}

There was no significant correlation between stem length and mycorrhizal colonisation (Spearman's Rank; $r=-0.07116$, d.f. $=30, \mathrm{p}>0.5$ ) or between root section number and mycorrhizal colonisation (Spearman's Rank; $r=0.21975$, d.f. $=30, p>0.5$ ). See figures $2 a$ and $2 b$. N.B. Root section number corresponds with depth in profile where root section one is from the top of the profile and root section two is from the bottom of the profile.

The root systems from the group of trees with above ground stem systems showed a wide range of recruitments from $41 \%$ (high) to $0 \%$ (failed recruitment) while the group of trees with no above ground growth displayed $0 \%$ (failed) recruitment on all samples (see figures $3 \mathrm{a}$ and $3 \mathrm{~b}$ ). The data were analysed for significant differences in colonisation using a Student's $\mathrm{T}$ Test, $\mathrm{t}=4.2085$, d.f. $=18, \mathrm{p}<0.001$. A significant difference in colonisation levels between the two groups of trees was observed.

\section{Discussion}

Statistical analysis of the data found that neither stem length nor depth in root-profile had a significant impact on the percentage colonisation of ectomycorrhiza (Figs. 2 and 3). We therefore suggest that observed differences in mycorrhizal colonisation were due to the presence or absence of photosynthetic apparatus in the trees. The absence of any mycorrhizal recruitment on trees which lacked an above-ground stem system and therefore sufficient photosynthetic apparatus clearly demonstrates the importance of photosynthetic compounds not only in the regulation of ectomycorrhizal associations but also in the initial recruitment of the mycorrhiza. This authenticates photosynthetically-induced signals as crucial for mycorrhizal attachment.

The most obvious candidates as regulators for this recruitment are sugars, due to their presence in the roots, intrinsic link with photosynthesis and essential role within the mycorrhizal mutualism. However, the normal root development of the mutant trees in this experiment suggests the presence of sugar stores in these individuals. Were the presence or absence of sugars the main driving force behind the association then the sugar stores within the mutant individuals should induce some level of ectomycorrhizal association. As we found however, this was not the case. There is clearly some secondary signalling triggered by the active process of photosynthesis rather than the passive presence of sugars within the root complex which is responsible for mycorrhizal association.

What remains unclear however is the exact mechanism of the plants' signalling within the rhizosphere. The signalling compounds present within the rhizosphere need to be identified; phytohormones within plants are important both in regulation of growth and development (Lambers et al. 1998) and as chemical messengers. Their concentrations are largely affected by exposure to 


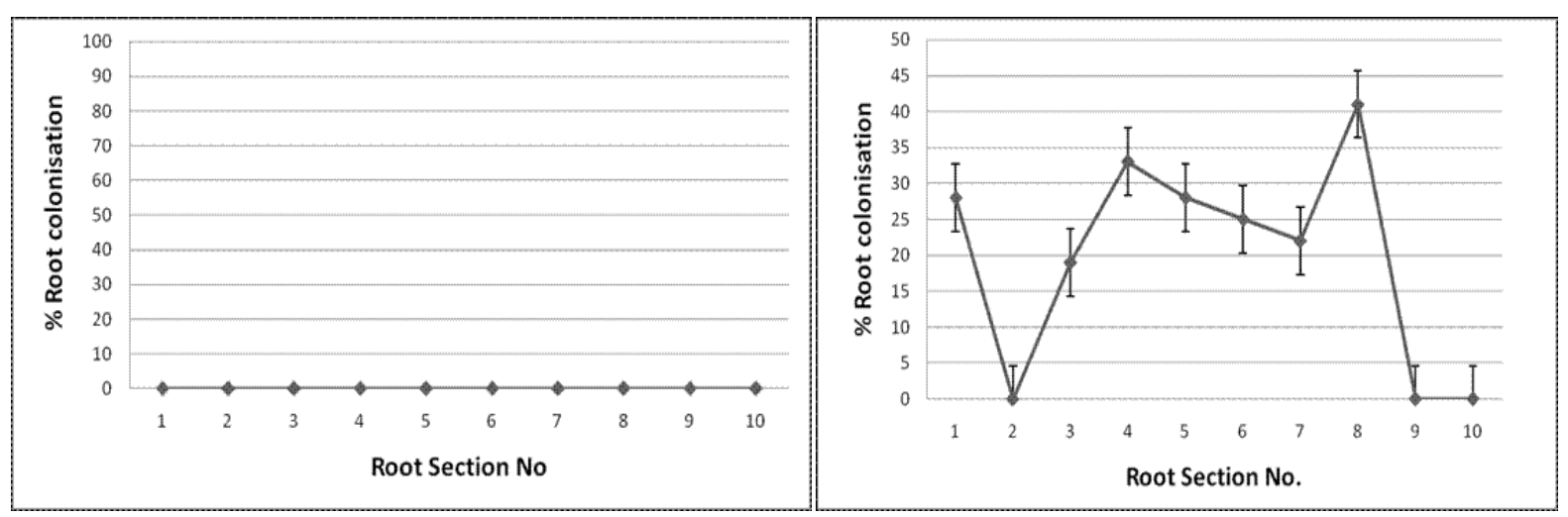

Fig. 3 - Comparison of \% mycorrhizal colonisation between the two groups. A. Percentage root colonisation $( \pm 1$ s.e.m.) at different depths in root profile from trees with above-ground growth, showing a range of recruitments from $41 \%$ root coverage (high) through to $0 \%$ coverage (failed recruitment). B. Percentage root colonisation $( \pm 1$ s.e.m.) at different depths in root profile from trees with no above ground growth, showing a complete failure of recruitment. N.B. Root section one is from the top of the profile and root section 10 is from the bottom of the profile.

certain environmental factors such as temperature and irradiance (Lambers et al. 1998) making them likely candidates as the compounds involved in photosynthetic signalling in response to the presence/absence of irradiance. The phytohormone particularly associated with responses to irradiance is indole-3-acetic acid, also known as IAA (Lambers et al. 1998, Kurepin et al. 2008).

The role of IAA in the ectomycorrhizal symbiosis is already well documented; the development of mycorrhiza on the root requires several morphological changes to the root structure including the reduction of tap root growth, stimulation of lateral root growth, changes to the shape of the epidermal root cells and the inhibition of root hair elongation (Martin et al. 1987, Barker et al. 1998). These changes are thought to be largely due to an influx of IAA from both the plant and the mycorrhiza (Barker \& Tagu 2000), thought based upon the hormone theory proposed by Slankis in 1973 which names phytohormones, IAA and ethylene primarily, as unique drivers of the differentiation process involved in mycorrhizal recruitment and development.

IAA is associated with growth towards light and it has been found that low levels of photosynthetically active radiation (PAR) increased production of IAA by two to three times in Stellaria longipes kept in the shade (Kurepin et al. 2008). It is possible then that the signalling cascade within the rhizosphere which leads to mycorrhizal recruitment is regulated by IAA levels. The antagonistic relationship between IAA and hypaphorine in the association of the ectomycorrhiza, as well as morphological changes in the roots, is also part of this signalling cascade. High levels of IAA could lead to the inhibition of the action of hypaphorine and therefore an absence of the important morphological changes required for ectomycorrhizal association. Only once the PAR is high enough to support ectomycorrhizal association will the IAA levels fall low enough for hypaphorine to take over and effect the necessary morphological changes required for the mycorrhizal association. However, more needs to be known about the relative effects of IAA and hypaphorine on the root morphology at the time of association before assumptions can be made on their relative roles in signalling cascades. Though there are currently no data regarding ectomycorrhiza, it is interesting to note that there are data linking IAA not only to communication and association but also to spore germination and hyphal elongation in vesicular arbuscular (endo-) mycorrhiza (Kaneko \& Tanimoto 2009).

There is still some way to go in unravelling the relationship between ectomycorrhiza and their plant associates. However we are coming closer to understanding the role of the nutrients and photosynthetically induced signalling compounds involved in the relationship beyond that simply of mutual exchange of resources. 
In this study, we have clearly demonstrated that plant signalling cascades are essential to their associations with ectomycorrhizal fungal mutualists, a group which includes some of the most valuable fungi in the world, truffles. The results of this study will not only have important implications for field studies into the ecological relationships formed by trees but will also advance the technology involved in cultivating these valuable fungi.

\section{Acknowledgements}

We thank Dr E. Thomas for proof reading and comments on the manuscript and E. BleaklyDean for assistance with data collection.

\section{References}

Barker SJ, Tagu D. 2000 - The roles of Auxins and cytokinins in mycorrhizal symbioses. Journal of Plant Growth Regulation. 19,144-154.

Barker SJ, Tagu D, Delp G. 1998 - Regulation of root and fungal morphogenesis in mycorrhizal symbioses. Plant Physiology 116, 1201-1207.

Béguiristain T, Cote R, Rubini P, Jay-Allemand C, Lapeyrie F. 1995 - Hypaphorine accumulation in the hyphae of the ectomycorrhizal fungus Pisolithus tinctorius. Phytochemistry 40, 10891091.

Bücking H, Heyser W. 2003 - Uptake and transfer of nutrients in ectomycorrhizal associations: interactions between photosynthesis and phosphate nutrition. Mycorrhiza 13, 59-68.

Ditengou FA, Lapeyrie F. 2000 - Hypaphorine from the ectomycorrhizal fungus Pisolithus tinctorius counteracts activities of indole-3-acetic acid and ethylene but not synthetic auxins in Eucalypt seedlings. Molecular Plant Microbe Interactions. 13, 151-158.

Ditengou FA, Raudaskoski M, Lapeyrie F. 2003.Hypaphorine, an indole-3-acetic acid antagonist delivered by the ectomycorrhizal fungus Pisolithus tinctorius, induces reorganisation of actin and the microtubule cytoskeleton in Eucalyptus globules ssp bicostata root hairs. Planta 218, $217-225$.

Gay G, Normand L, Marmeisse R, Sotta B, Debaud JC. 1994 - Auxin over producer mutants of Hebeloma cylindrosporum Romagnesi have increased mycorrhizal activity. New Phytologist. 128, 645-657.

Kaneko M, Tanimoto E. 2009 - IAA-regulation of hyphal elongation and spore germination in arbuscular mycorrhizal fungus, Gigaspora margarit. International Symposium "Root Research and Applications".

Kurepin LV, Emery RJN, Chinnappa CC, Reid DM. 2008 - Light irradiance differentially regulates endogenous levels of cytokinins and IAA in alpine and prairie genotypes of Stellaria longipes. Physiologia Plantarum 134, 624-635.

Lambers H, Chapin III FS, Pons TL 1998 - Plant Physiological Ecology. Springer, New York.

Martin F, Ramstedt M, Söderhäll K. 1987 - Carbon and nitrogen metabolism in ectomycorrhizal fungi and ectomycorrhizas. Biochimie 69, 569-581.

Nehls U. 2008 - Mastering ectomycorrhizal symbiosis: the impact of carbohydrates. Journal of Experimental Botany. 59, 1097-1108.

Palmer JG, Hacskaylo E. 1970 - Ectomycorrhizal fungi in pure culture I. Growth on single carbon sources. Physiologia Plantarum. 23, 1187-1197.

Peterson RL, Bonfante P 1994 - Comparative structure of vesicular-arbuscular mycorrhizas and ectomycorrhizas. Plant and Soil 159, 79-88.

Reboutier D. 2002 - The indolic compound hypaphorine produced by ectomycorrhizal fungus interferes with auxin action and evolves early responses in nonhost Arabidopsis thaliana. Molecular Plant Microbe Interactions 15, 932-938.

Regvar M, Gogala N. 1996 - Changes in root growth patterns of (Picea abies) spruce roots by inoculation with an ectomycorrhizal fungus Pisolithus tinctorius and jasmonic acid treatment. Trees 10, 410-414. 
Salzer P, Hager A. 1991 - Sucrose utilization of the ectomycorrhizal fungi Amanita muscaria and Hebeloma crustuliniforme depends on the cell wall-bound invertase activity of their host Picea abies. Botanica Acta 104, 439-445.

Slankis V. 1973 - Hormonal relationships in mycorrhizal development. In: Marks GC, Kozlowski TT (eds) Ectomycorrhizae: their ecology and physiology. Academic Press, New York. p 231298.

Smith SE, Gianinazzi-Pearson V, Koide R, Cairney JWG. 1994 - Nutrient transport in mycorrhizas: structure, physiology and consequences for efficiency of the symbiosis. Plant Soil 159, 103-113.

Son CL, Smith SE. 1988 - Mycorrhizal growth response: interactions between photon irradiance and phosphorus nutrition. New Phytologist 108, 305-314.

Wang B, Qiu YL. 2006 - Phylogenetic distribution and evolution of mycorrhizas in land plants. Mycorrhiza 16(5), 299-363. 